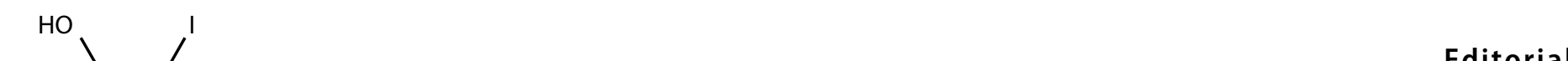

\section{Hormone in Zahlen}

- Ca. 1000 verschiedene Hormone existieren nach Vermutungen von Wissenschaftlern. Ungefähr 100 der Botenstoffe sind derzeit bekannt.

- Ein Drittel aller Deutschen hat eine Störung der Schilddrüsenfunktion.

- Vor 150 Jahren waren Mädchen bei ihrer ersten Regelblutung durchschnittlich 17 Jahre alt. Heute sind sie 13 Jahre - ein Grund: Sie werden immer dicker. Adipositas führt $\mathrm{zu}$ einem früheren Eintritt der Menarche.

- 4 von 5 Befragten sind nicht bereit Hormone zu nehmen, um den Alterungsprozess aufzuhalten.

- Der Melatoninspiegel ist im Winter $\mathbf{8 0 \%}$ höher als im lichtreichen Sommer.

planet-wissen.de, schilddruesenliga.de, wdr.de, statista.de, focus.de

\section{Tückisches Flimmern aus der Drüse}

Nicht nur Patienten mit einer Hyperthyreose haben ein erhöhtes Risiko für Vorhofflimmern, sondern bereits Patienten mit subklinischer Schilddrüsenüberfunktion oder mit einer hochnormalen Euthyreose. Die Assoziation entdeckten dänische Endokrinologen und Kardiologen bei der Auswertung der Daten von mehr als einer halben Million Patienten. Die statistische Auswertung ergab, dass die Wahrscheinlichkeit für Vorhofflimmern bei Patienten mit normalen T4Werten und verringertem TSH sowie hochnormaler Schilddrüsenfunktion im Vergleich zu Patienten mit euthyreoter Schilddrüse um etwa $10 \%$ erhöht war.

BMJ 2012, 345:e7895

\section{Zu viel Mann, zu wenig Mann}

Frauen mit Bärten, Männer mit Brüsten - ganz schön grausam, wie Hormone zuschlagen können. Doch müssen derart Geplagte ihr Leben zum Glück nicht mehr im Wanderzirkus fristen! Neben Schere und FdH gibt's allerlei Möglichkeiten, unerwünschte Hormonwirkungen einzudämmen. Wie man zeitgerecht und effektiv vorgeht, erfahren Sie in unserem Schwerpunkt „Hormone“.

Dr. Sonja Kempinski Chefredakteurin

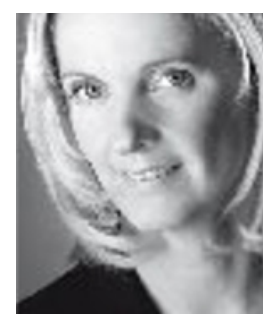

\title{
Adipositas lässt Nasen triefen
}

Aktuelle Erkenntnisse lassen vermuten, dass sowohl die chronische Rhinosinusitis (CRS) und die allergische Rhinitis (AR) als auch die Adipositas mit entzündlichen Prozessen einhergehen. In einer amerikanischen Studie der Harvard Medical School wurde nun untersucht, ob zwischen krankhaftem Übergewicht und sinunasalen Entzündungen ein Zusammenhang besteht. Mittels Querschnittsanalyse einer amerikanischen Panelbefragung aus den Jahren 2008 und 2010 wurden Erwachsene mit chronischer CRS und/oder AR erfasst, gleichzeitig wurde das Körpergewicht der
Personen ermittelt. Das Ergebnis: 7,7\% der erwachsenen Amerikaner litten an einer allergischen Rhinitis, 5,7\% an einer chronischen Rhinosinusitis. Fast ein Drittel galt als adipös. Mit zunehmendem Körpergewicht wurde der Zusammenhang zwischen AR bzw. CRS und Adipositas immer deutlicher. In der Analyse lag bei den adipösen Probanden der Anteil der Personen mit allergischer Rhinitis um 22\% und mit chronischer Rhinosinusitis um $31 \%$ höher als in der gesamten Studienpopulation.

Bhattacharyya N, Laryngoscope 2013 (online first)
Eier suchen und gewinnen!

Der Startschuss für die große

Springer Medizin Eiersuche ist gefallen.

Gehen Sie online - und finden Sie die Ostereier, die das Lösungswort verraten! Mit ein bisschen Glück gewinnen Sie einen der tollen Preise.

Die Eiersuche und alle weiteren Informationen finden Sie unter: - www.springermedizin.de/ osterquiz-2013

\section{Sildenafil bei ED: Mit Testosteron noch standfester?}

Männer mit niedrigen Hormonspiegeln, die Sildenafil einnehmen, können ihre Erektionsfähigkeit durch die zusätzliche Zufuhr von Testosteron nicht weiter verbessern - so das Ergebnis einer US-Studie. Die Autoren wollten wissen, ob die transdermale Zufuhr von Testosteron, zusätzlich zu einer bestehenden Sildenafil-Therapie, die erektile Dysfunktion bei Männern mit einem Gesamttestosteron $<11,45 \mathrm{nmol} / 1$ und einem freien Testosteron $<173,35 \mathrm{pmol} / \mathrm{l}$ weiter verbessern kann. In eine randomisierte, placebokontrollierte Doppelblind-Studie schlossen sie 140 Männer zwischen 40 und 70 Jahren ein. Die zu Beginn der Studie ähnlichen Scores der erektilen Dysfunktion (EFDScore) wurden in beiden Gruppen durch die Einnahme von Sildenafil ähnlich verbessert, aber der erhoffte Effekt auf eine bessere Erektionsfähigkeit durch zusätzliche Testosteronzufuhr blieb aus.

(st) 